\title{
ON BOUNDARY CONDITIONS FOR SYMMETRIC SUBMARKOVIAN RESOLVENTS
}

\author{
BY JOANNE ELLIOTT AND MARTIN R. SILVERSTEIN
}

Communicated by H. P. McKean, February 26, 1970

1. Introduction. In a recent paper [4], M. Fukushima has established a one-to-one correspondence between symmetric markovian semigroups which satisfy the heat equation on a bounded domain $D$ in Euclidean $n$-space and certain Dirichlet spaces on the Martin boundary of that domain. In this note we give an extension of his result to a much more general context.

Fukushima considers semigroups with resolvent kernels of the form

$$
G_{\alpha}(x, y)=G_{\alpha}^{0}(x, y)+R_{\alpha}(x, y)
$$

where $G_{\alpha}^{0}$ is the "absorbing barrier" or minimal resolvent for Brownian motion on $D$ and $R_{\alpha}(x, y)$, defined for $x$ and $y$ in $D$, is a nonnegative, symmetric " $\alpha$-harmonic" term, i.e. $R_{\alpha}$ satisfies the equation $\alpha R_{\alpha}$ $-(1 / 2) \Delta R_{\alpha}=0$ in $D$ as a function of $x$ for fixed $y$. Also, it is assumed that $\alpha G_{\alpha} 1=1$ in $D$. We start with a given nonnegative symmetric resolvent $G_{\alpha}^{0}$ which is submarkovian, i.e. $\alpha G_{\alpha}^{0} 1 \leqq 1$, and then consider resolvents $G_{\alpha} \geqq G_{\alpha}^{0}$ which are symmetric and submarkovian. The Laplacian operator which plays a central role in Fukushima's work is here replaced by a much more general type of operator $A$ which may not even be a local operator. The main results will be found in Theorems 1-3. Our method of proof is different from that of Fukushima. The details will be published elsewhere.

2. Preliminaries. Let $(X, d x)$ be a sigma finite measure space and let $(,)_{X}$ or $(,)_{d x, X}$ denote the standard inner product on $L^{2}(X)$, the Hilbert space of real-valued square integrable functions on $X$.

2.1. Definition. A symmetric submarkovian resolvent on $L^{2}(X)$ is a family $\left\{G_{\alpha}, \alpha>0\right\}$ of bounded linear operators on $L^{2}(X)$ such that

2.1.1. $G_{\alpha} f \geqq 0$ a.e. whenever $f \geqq 0$ a.e. and $\alpha G_{\alpha} 1 \leqq 1$ a.e.

2.1.2. $G_{\alpha}-G_{\beta}=(\beta-\alpha) G_{\alpha} G_{\beta}$.

2.2. Definition. The measurable function $g$ is a normalized contraction of the measurable function $f$ if $|g(x)| \leqq|f(x)|$ and $|g(x)-g(y)| \leqq|f(x)-f(y)|$ for all $x, y$ in $X$.

2.3. Definition. A Dirichlet space relative to $L^{2}(X)$ is a pair $(F, \varepsilon)$ where

AMS Subject Classifications. Primary 4615; Secondary 6060.

Key Words and Phrases. Submarkovian resolvents, boundary conditions, Dirichlet spaces, stochastic processes. 
2.3.1. $F$ is a linear (but not necessarily closed) subset of $L^{2}(X)$ and $\varepsilon$ is a positive semidefinite symmetric bilinear form on $F$.

2.3.2. For each $\alpha>0$ the linear space $F$ is a Hilbert space relative to the inner product

$$
\varepsilon_{\alpha}(f, g)=\varepsilon(f, g)+\alpha(f, g)_{X} .
$$

2.3.3. If $f \in F$ and if $g$ is a normalized contraction of $f$, then $g \in F$ and $\mathcal{E}(g, g) \leqq \mathcal{E}(f, f)$.

The connection between Dirichlet spaces and resolvents may be summed up as follows. Condition 2.1.2 guarantees that the $G_{\alpha}$ form a commuting family of bounded, symmetric operators in $L^{2}(X)$ and so the spectral theory can be applied to establish the existence of a negative definite operator $A$, the so-called generator of the resolvent family $\left\{G_{\alpha}, \alpha>0\right\}$, which is selfadjoint as an operator in the Hilbert space $\bar{R}$, the closure of the common range $R$ of the $G_{\alpha}$. Also $(\alpha-A) G_{\alpha} f$ $=f$ for $f \in \bar{R}$, and $G_{\alpha}(\alpha-A) f=f$ for $f \in R$. Then $F$ is just the domain of the unique positive square root $\sqrt{ }(-A)$ of $A$ and $\mathcal{E}(f, f)$ $=(\sqrt{ }(-A) f, \sqrt{ }(-A) f)_{X}$ for $f \in F$. It follows easily that

$$
\mathcal{E}(f, f)=\operatorname{Lim} \alpha\left(f-\alpha G_{\alpha} f, f\right)_{X} \quad(\alpha \uparrow \infty) .
$$

The submarkovian property of the operators $G_{\alpha}$ is equivalent to the contraction property for the Dirichlet space $(F, \varepsilon)$.

3. The general set-up. Let $(D, d x)$ and $(M, d \zeta)$ be sigma finite measure spaces with the possibility that $d \zeta$ vanishes identically. Let $\Omega=D \cup M$. We suppose given once and for all a symmetric submarkovian resolvent $\left\{G_{\alpha}^{0}: \alpha>0\right\}$ on $L^{2}(D)$ which has been regularized in the following sense. Measures $G_{\alpha}^{0}(x, d \cdot)$ on $D$ have been selected so that a representative for $G_{\alpha}^{0} f$ is defined by the action of these measures, viz.

$$
G_{\alpha}^{0} f(x)=\int_{D} G_{\alpha}^{0}(x, d y) f(y)
$$

and so with this choice of a representative the conditions 2.1 .1 and 2.1.2 hold identically on $D$ whenever they make sense. We assume further that 1 is excessive (that is, $\alpha G_{\alpha}^{0} 1$ increases to 1 identically as $\alpha$ increases to $\infty$ ), and we define $h_{a}$, the active part of 1 , and $h_{p}$, the passive part of 1 , by

$$
h_{p}=\operatorname{Lim}_{\alpha \downarrow 0} \alpha G_{\alpha}^{0} 1 \quad h_{a}=1-h_{p} .
$$

(See $[3, \S 7]$ where the significance of $h_{a}$ and $h_{p}$ is discussed.) Our basic hypothesis is that $h_{a}$ can be represented as 


$$
h_{a}(x)=\int_{M} K(x, \xi) d \mu(\xi)
$$

where $d \mu$ is a bounded measure on $M$ such that $d \zeta$ is absolutely continuous with respect to $d \mu$ and where $K(x, \xi)$ is a positive kernel defined and jointly measurable on $D \times M$ and satisfying:

3.4.1. For each $\xi$ in $M$ the function $K(\cdot, \xi)$ is excessive relative to the $G_{\alpha}^{0}$.

3.4.2. For each $x$ in $D$ the function $K(x, \cdot)$ is bounded and bounded away from 0 on $M$.

3.4.3. If $\phi \in L^{1}(d \mu)$ and if both $\int_{M} K(\cdot, \xi) \phi(\xi) d \mu(\xi)=0$ (a.e. $d x$ ) on $D$ and $\phi=0$ (a.e. $d \zeta$ ) on $M$, then $\phi=0$ (a.e. $d \mu$ ).

In addition we assume

3.4.4. There exists a nonzero excessive function $r$ belonging to $L^{2}(D)$. (Since 1 is excessive, this assumption is superfluous when $D$ has finite $d x$ measure.)

For $\alpha>0$ the auxiliary kernel $K_{\alpha}(x, \xi)$ on $D \times M$ is defined by

$$
K_{\alpha}(x, \xi)=K(x, \xi)-\alpha \int_{D} G_{\alpha}^{0}(x, d y) K(y, \xi) .
$$

For $\alpha \geqq 0$ and for $\phi$ in $L^{1}(d \mu)$, the function $H_{\alpha} \phi$ on $\Omega$ is defined by

$$
\begin{aligned}
H_{\alpha} \phi(x) & =\int_{M} K_{\alpha}(x, \xi) \phi(\xi) d \mu(\xi) & & x \in D, \\
& =\phi(x) & & x \in M .
\end{aligned}
$$

For $\alpha \geqq 0$ and for $f$ on $\Omega$ we define

$$
\hat{H}_{\alpha} f(\xi)=\int_{D} f(x) K_{\alpha}(x, \xi) d x+\frac{d \xi}{d \mu}(\xi) f(\xi) \quad(\xi \in M) .
$$

For $\xi, \eta \in M$, for $\phi, \psi$ defined on $M$, and for $0 \leqq \beta \leqq \alpha$ we introduce

$$
\begin{aligned}
U_{\beta, \alpha}^{0}(\xi, \eta) & =(\alpha-\beta) \int_{D} d x K_{\alpha}(x, \xi) K_{\alpha}(x, \eta), \\
U_{\beta, \alpha} \phi(\xi) & =\int_{M} U_{\beta, \alpha}^{0}(\xi, \eta) \phi(\eta) d \mu(\eta)+(\alpha-\beta) \frac{d \xi}{d \mu}(\xi) \phi(\xi), \\
U_{\beta, \alpha}^{0}\langle\phi, \psi\rangle & =\int_{M} d \mu(\eta) \int_{M} d \mu(\xi)\{\phi(\xi)-\phi(\eta)\}\{\psi(\xi)-\psi(\eta)\} U_{\beta, \alpha}^{0}(\xi, \eta), \\
U_{\beta, \alpha}(\phi, \psi) & =\int_{M} d \mu(\xi) \phi(\xi) U_{\beta, \alpha} \psi(\xi), \\
p(\xi) & =\hat{H}_{1} h_{p}(\xi) .
\end{aligned}
$$


It is easy to check that

$$
\begin{array}{ll}
U_{\beta, \gamma}(\phi, \psi)=U_{\beta, \alpha}(\phi, \psi)+U_{\alpha, \gamma}(\phi, \psi), & 0 \leqq \beta<\alpha<\gamma, \\
U_{\beta, \gamma}^{0}\langle\phi, \psi\rangle=U_{\beta, \alpha}^{0}\langle\phi, \psi\rangle+U_{\alpha, \gamma}^{0}\langle\phi, \psi\rangle, & 0 \leqq \beta<\alpha<\gamma .
\end{array}
$$

The last two relations permit us to introduce

$$
\begin{aligned}
U_{\beta, \infty}^{0}(\xi, \eta) & =\operatorname{Lim} U_{\beta, \alpha}^{0}(\xi, \eta) & & (\alpha \uparrow \infty), \\
U_{\beta, \infty}^{0}\langle\phi, \psi\rangle & =\operatorname{Lim} U_{\beta, \alpha}^{0}\langle\phi, \psi\rangle & & (\alpha \uparrow \infty) .
\end{aligned}
$$

Finally, we introduce the measure $d \mu^{1}(\xi)=\hat{H}_{1} h_{a}(\xi) d \mu(\xi)$ on $M$ and the pair $\left(H^{R}, \mathcal{E}^{R}\right)$ viz.

$$
\begin{aligned}
H^{R} & =\left\{\phi \in L^{2}\left(d \mu^{1}\right): \mathcal{E}^{R}(\phi, \phi)<\infty\right\}, \\
\mathcal{E}^{R}(\phi, \psi) & =\frac{1}{2} U_{0, \infty}^{0}\langle\phi, \psi\rangle+\int_{M} d \mu(\xi) p(\xi) \phi(\xi) \psi(\xi) .
\end{aligned}
$$

It is easily checked that $\left(H^{R}, \mathcal{E}^{R}\right)$ is a Dirichlet space relative to $L^{2}\left(d \mu^{1}, M\right)$. (The significance of the superscript $R$ will be explained after Theorem 2 in $\$ 4$.)

4. The main result. A submarkovian resolvent $\left\{G_{\alpha}: \alpha>0\right\}$, symmetric relative to $d x+d \zeta$ on $\Omega$, will be called admissible if

4.1.1. For $f \geqq 0$ on $\Omega$, a version for $G_{\alpha} f$ has been selected which is defined identically on $D$ and (a.e. $d \mu$ ) on $M$ and such that 2.1.1 and 2.1.2 are valid identically on $D$ and (a.e. $d \mu$ ) on $M$.

4.1.2. $G_{\alpha} f=G_{\alpha}^{0}+H_{\alpha} G_{\alpha} f$ identically on $D$ for $\alpha>0$.

4.2 Convention. The resolvent $\left\{G_{\alpha}^{0}: \alpha>0\right\}$ is regarded as acting on functions on $\Omega$ in the obvious way: $G_{\alpha}^{0} f=0$ on $M$ and $G_{\alpha}^{0} f=G_{\alpha}^{0} f^{\prime}$ on $D$ where $f^{\prime}$ is the restriction of $f$ to $D$. Then $G_{\alpha}^{0}$ is an admissible resolvent, and 4.1.2 is now true not only on $D$ but on all of $\Omega$.

Given any admissible resolvent $\left\{G_{\alpha}: \alpha>0\right\}$ we denote the associated generator by $A$ and the associated Dirichlet space norms by $\mathcal{E}$ and $\mathcal{E}_{\alpha}$.

THEOREM 1. If $\left\{G_{\alpha}: \alpha>0\right\}$ is an admissible resolvent on $\Omega$, then there is a unique pair $\left(H^{M}, \varepsilon^{M}\right)$ where

(i) $H^{M}$ is a linear subset of $H^{R}$ which is stable with respect to normalized contractions.

(ii) $\mathcal{E}^{M}$ is a bilinear form on $H^{M}$ which dominates $\mathcal{E}^{R}$ in the following sense: if $\phi$ is in $H^{M}$ and if $\psi$ is a normalized contraction of $\phi$, then

$$
0 \leqq \mathcal{E}^{M}(\psi, \psi)-\mathcal{E}^{R}(\psi, \psi) \leqq \mathcal{E}^{M}(\phi, \phi)-\mathcal{E}^{R}(\phi, \phi) .
$$

(iii) The pair $\left(H^{M}, \varepsilon^{M}\right)$ is a Dirichlet space relative to $L^{2}\left(d \mu^{1}\right)$. 
(iv) For each $\alpha>0$ the operator $H_{\alpha}$ maps $H^{M}$ into the domain of $\sqrt{ }(-A)$ and for $\phi$ in $H^{M}$

$$
\varepsilon_{\alpha}\left(H_{\alpha} \phi, H_{\alpha} \phi\right)=\mathcal{E}^{M}(\phi, \phi)+U_{0, \alpha}(\phi, \phi) .
$$

Moreover, domain $\sqrt{ }\left(-A^{0}\right)$ and the closure of $H_{\alpha}\left(H^{M}\right)$ are complementary orthogonal subspaces in domain $\sqrt{ }(-A)$ relative to the inner product $\varepsilon_{\alpha}$.

Conversely, to every pair $\left(H^{M}, \varepsilon^{M}\right)$ satisfying (i), (ii), and (iii) there is associated a unique admissible resolvent such that (iv) is valid.

Theorem 1 yields a complete characterization of admissible resolvents $\left\{G_{\alpha}: \alpha>0\right\}$ by means of Dirichlet spaces $\left(H^{M}, \varepsilon^{M}\right)$ on $M$. The next theorem gives additional information on the connection between $\left(H^{M}, \varepsilon^{M}\right)$ and $\left\{G_{\alpha}: \alpha>0\right\}$.

Theorem 2. Let $\left\{G_{\alpha}: \alpha>0\right\}$ and $\left(H^{M}, \mathcal{E}^{M}\right)$ be as in Theorem 1 and for $\alpha>0$, and for $\phi, \psi$ in $H^{M}$ let

$$
\varepsilon_{\alpha}^{M}(\phi, \psi)=\varepsilon^{M}(\phi, \psi)+U_{0, \alpha}(\phi, \psi) .
$$

Then

(i) For each $\alpha>0$ the pair $\left(H^{M}, \varepsilon_{\alpha}^{M}\right)$ is a Dirichlet space relative to $L^{2}(d \mu)$.

(ii) If $\left\{\widetilde{R}_{\alpha ; \lambda}: \lambda>0\right\}$ is the resolvent on $L^{2}(d \mu)$ corresponding to the Dirichlet space $\left(H^{M}, \varepsilon_{\alpha}^{M}\right)$, then $\widetilde{R}_{\alpha}=\operatorname{Lim} \widetilde{R}_{\alpha ; \lambda}(\lambda \downarrow 0)$ exists in the strong operator topology on $L^{2}(d \mu)$, and for $f$ on $\Omega$

$$
G_{\alpha} f=G_{\alpha}^{0} f+H_{\alpha} \widetilde{R}_{\alpha} \hat{H}_{\alpha} f
$$

REMARK. The resolvent $\left\{G_{\alpha}^{0}: \alpha>0\right\}$ corresponds to the absorbing barrier resolvent in [4] and to the minimal resolvent in [1], [2], and [3]. The Dirichlet space $\left(H^{R}, \varepsilon^{R}\right)$ is the analogue of the boundary Dirichlet space associate in [4] with the reflecting barrier process (hence the superscript $R$ ). Just as in [4] it will turn out that the relevant Dirichlet spaces on the boundary are just those contained in $\left(H^{R}, \mathcal{E}^{R}\right)$ in an appropriate sense.

To formulate the results in terms of boundary conditions we introduce the following two operators:

4.3. $F$ defined on $\Omega$ is in the domain of the local 1-generator $A_{1}$ if $F$ is in the domain of $\sqrt{ }\left(-A^{R}\right)$ and if there exists $f$ in $L^{2}(D)$ such that $\varepsilon_{1}^{R}(F, g)=-\int_{D} d x f(x) g(x)$ for $g$ in the domain of $\sqrt{ }\left(-A^{0}\right)$. In this case $A_{1} F=f$. (Here $A^{0}$ is the absorbing barrier generator and $A^{R}$ the reflecting barrier generator. It follows from Theorem 1 (iv) that

$$
\text { domain } \sqrt{ }\left(-A^{0}\right) \subset \text { domain } \sqrt{ }(-A) \subset \text { domain } \sqrt{ }\left(-A^{R}\right)
$$

for $A$ the generator of an admissible resolvent.) 
4.4. $F$ defined on $\Omega$ is in the domain of $A_{1}^{+}$, the extended 1-generator if $F$ is in the domain of the local 1-generator $A_{1}$, if the boundary value $\gamma F$ of $F$ belongs to $H^{M}$ and if there exists $\phi$ in $L^{2}(M, d \zeta)$ such that

$$
\varepsilon_{1}^{M}(\gamma F, \psi)+\int_{M} d \mu(\xi) \psi(\xi) \hat{H}_{1} A_{1} F(\xi)=\int_{M} d \zeta(\xi) \psi(\xi) \phi(\xi)
$$

for all $\psi$ in $H^{M}$. In this case

$$
\begin{aligned}
A_{1}{ }_{1}^{+} F & =A_{1} F & & \text { on } D, \\
& =\phi & & \text { on } M .
\end{aligned}
$$

(In the course of proving Theorem 1 we show that the boundary value $\gamma F$ of $F$ is well defined as an element of $L^{1}(M, d \mu)$ for $F$ in domain $\sqrt{ }\left(-A^{R}\right)$ and in particular for $F$ in domain $A_{1}$.) Then we have

THEOREM 3. Let $A$ be the generator of the admissible resolvent $\left\{G_{\alpha}: \alpha>0\right\}$.

(i) If $d \zeta=0$, then $F$ is in the domain of $A$ if and only if $F$ is in the domain of the local 1-generator $A_{1}$ and

$$
\varepsilon_{1}^{M}(\gamma F, \psi)+\int_{M} d \mu(\xi) \psi(\xi) \hat{H}_{1} A_{1} F(\xi)=0
$$

for all $\psi$ in $H^{M}$. In this case $A F=A_{1} F+F$.

(ii) If $d \zeta \neq 0$, then $F$ is in the domain of $A$ if and only if $F$ is in the domain of the extended 1-generator $A_{1}^{+}$and then $A F=A_{1}^{+} F+F$.

REMARK. Concerning the interpretation of the Dirichlet space $\left(H^{M}, \mathcal{E}^{M}\right)$ in terms of "Markov processes on the boundary" we refer the reader to [5] and [6]. It remains to be established to what extent or in what sense this interpretation is valid in the present context.

\section{REFERENCES}

1. J. Elliott, Dirichlet spaces associated with integro-differential operators. I, Illinois J. Math. 9 (1965), 87-98. MR 30 \#5159.

2. - Lateral conditions for semigroups involving mappings in $L^{p} . \mathrm{I}, \mathrm{J}$. Math. Anal. Appl. 25 (1969), 388-410. MR 39 \#3350.

3. W. Feller, On boundaries and lateral conditions for the Kolmogorov differential equations, Ann. of Math. (2) 65 (1957), 527-570. MR 19, 892.

4. M. Fukushima, On boundary conditions for multi-dimensional Brownian motions with symmetric resolvent densities, J. Math. Soc. Japan 21 (1969), 58-93. MR 38 \#5291.

5. K. Sato, A decomposition of Markov processes, J. Math. Soc. Japan 17 (1965), 269-293. MR 31 \#6284.

6. K. Sato and T. Ueno, Multi-dimensional diffusion and the Markov process on the boundary, J. Math. Kyoto Univ. 4 (1964/65), 529-605. MR 33 \#6702.

Rutgers University, New Brunswick, New Jersey 08903 